\title{
Diseño de molde para el establecimiento de la relación entre resultados de análisis térmico y microestructura del aluminio A357 en moldeo por microfusión
}

\author{
Y. Bilbao*, M. Espinosa**, G. Artola**, A.I. Fernández-Calvo*** y P.P. Rodríguez*
}

\begin{abstract}
Resumen El tratamiento del metal fundido resulta esencial para mejorar las propiedades mecánicas de las aleaciones de aluminio. Los métodos de análisis térmico empleados habitualmente como medio de control están basados en el enfriamiento del metal en una copa metálica o en arena. En el caso de la microfusión, el enfriamiento se produce en un molde refractario a alta temperatura (entre 400 y $700^{\circ} \mathrm{C}$ ), en el que las piezas además poseen espesores muy finos, de modo que los resultados de análisis térmico no son del todo fieles a lo que se obtiene en pieza. Como resultado del presente trabajo se obtiene un diseño de molde capaz de ajustarse a la tipología de pieza común en el moldeo a la cera perdida, lo que permitirá establecer, en ensayos futuros, la relación entre los resultados de análisis térmico, la microestructura en pieza y las propiedades mecánicas finales.
\end{abstract}

\section{Design of a mould for the study of the relationship between thermal analysis results and microstructure of aluminium alloy A357 in investment casting}

\begin{abstract}
Melt treatment of aluminium alloys is essential for optimizing their mechanical properties. Thermal analysis methods usually used as control are based on the cooling of the metal in metallic or sand cups. In investment casting, however, cooling takes place in a refractory mould at high temperatures $\left(400-700^{\circ} \mathrm{C}\right)$, where parts are characterized by thin thicknesses, so the results given by thermal analysis devices are not really representative of what happens in the parts. As a result of the present work a mould design is obtained, which adjusts itself to the typology of the parts in investment casting, and will make it possible to establish, in future trials, a relationship between thermal analysis results, microstructure in the part and final mechanical properties.
\end{abstract}

Keywords

Investment casting; Aluminium alloys; Mould design; Mechanical optimization.

\section{INTRODUCCIÓN}

El sector aeronáutico requiere, habitualmente, de piezas ligeras de geometría compleja y paredes muy delgadas. En estos casos, el moldeo a la cera perdida (o microfusión) de aleaciones de aluminio es el medio ideal para su fabricación. Así, "Microfusión de Aluminio, S. A." produce piezas en aleaciones A356 y A357, caracterizadas por una muy buena colabilidad. En la mayoría de los casos, las exigencias de los clientes requieren, además, buenas propiedades mecánicas y para conseguirlas, las propiedades del metal fundido resultan esenciales.

En el ámbito industrial, los medios de control habituales son el análisis químico y el térmico. Este último permite obtener una predicción de la microestructura resultante, basándose en el enfriamiento del metal en una copa estándar, generalmente, metálica o en arena ${ }^{[1]}$. Se pueden conocer, de este modo, tanto el afino del metal como su grado de modificado. Ambas características resultan determinantes en las propiedades mecánicas: un correcto afino de grano es capaz de aumentar la resistencia y el alargamiento del metal,

\footnotetext{
* ALFA IDEI A.I.E (Grupo ALFA), Avda. Otaola 4,20600 Eibar.

** MICROFUSIÓN DE ALUMINIO, S. A. (Grupo ALFA), Torrekua 3, 20600 Eibar.

${ }^{* * *}$ AZTERLAN, Ingeniería y Procesos de Fundición, Aliendalde Auzunea 6, 48200 Durango.
} 
mientras que el modificado de la fase eutéctica mejora las propiedades, tanto de fatiga como de alargamiento, así como la maquinabilidad. La distribución de la porosidad se ve igualmente mejorada ${ }^{[2]}$.

Sin embargo, el enfriamiento, en dicha copa estándar, no se asemeja al que se produce en la pieza de microfusión, donde el molde es refractario y se halla a temperaturas entre 400 y $700{ }^{\circ} \mathrm{C}$ (Fig. 1). De hecho, la solidificación de la pieza dependerá de las propiedades de la cáscara cerámica, el espesor de la pieza, su orientación y su posición en el molde. La dependencia de las propiedades mecánicas con la composición química y las condiciones de enfriamiento ha sido observada en numerosos estudios ${ }^{[3-5]}$.

Con el fin de conseguir una equivalencia entre la predicción del equipo de análisis térmico y la microestructura en la pieza y tener capacidad, así, de optimizar el tratamiento del caldo y, con ello, las propiedades mecánicas finales, se diseña una probeta y dos moldes para la realización de ensayos preliminares, cuyos resultados permitirán ajustar el diseño para pruebas futuras.

\section{MÉTODO EXPERIMENTAL}

\subsection{Diseño de molde y probeta}

Se diseña una probeta que consta de tres escalones de diferente grosor y área lo suficientemente grande

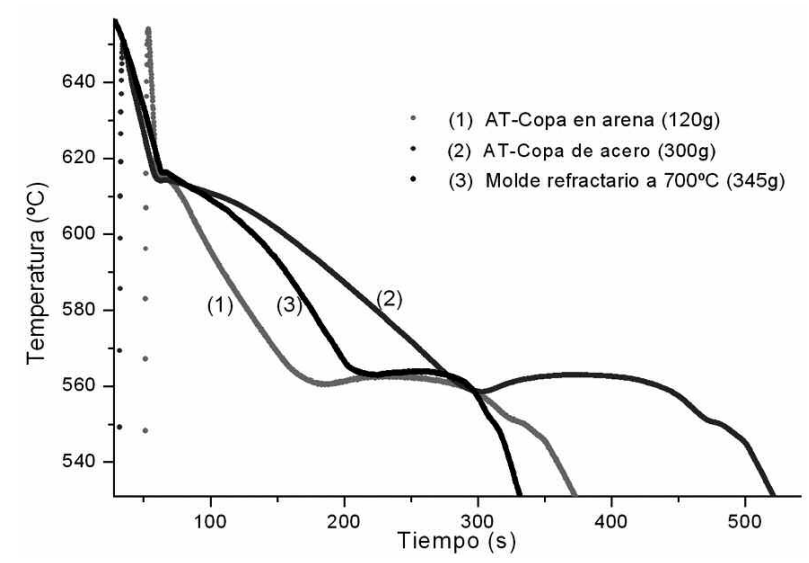

Figura 1. Curvas experimentales de enfriamiento, en copas estándar de análisis térmico (AT). (1) $y(2)$, frente a pieza de microfusión (3). Se incluyen las masas de las copas y de la pieza de microfusión de aluminio.

Figure 1. Experimental cooling curves in thermal analysis cups $(A T)(1,2)$ versus investment cast part (3). Mass values of cups and aluminium casting are included. como para permitir la obtención de muestras. El diseño de la probeta está basado en los grosores característicos de la tipología de pieza de "Microfusión de Aluminio, S. A.”. En general, se trata de piezas con paredes delgadas, entre 2 y $4 \mathrm{~mm}$, si bien la presencia de bridas y nervios hace que existan secciones de mayor grosor. Así, se opta por escoger grosores extremos $(1,5$ y $16 \mathrm{~mm})$ junto a uno intermedio $(8 \mathrm{~mm})$ (Fig. 2).

En el diseño del molde se tienen en cuenta los siguientes factores: orientación de la probeta escalón (horizontal o vertical), posición en el molde (en ramas exteriores o en interiores) y proximidad al filtro (llenado, más o menos rápido). La ubicación en ramas interiores, así como la cercanía al filtro influirán, aumentando la radiación de calor en las piezas y provocando un enfriamiento más lento. Los moldes realizados se muestran en la figura 3 a) y b).

El molde I posee cuatro probetas en posición horizontal, dos en ramas interiores y dos en exteriores. Además, dos de ellas (una interior y una exterior) poseen su escalón más grueso cerca del filtro. En cuanto al molde II, se emplean seis probetas en posición vertical, siendo cuatro de ellas exteriores y dos interiores. En este caso también resulta importante la posición superior o inferior de los escalones fino y grueso.

\subsection{Procedimiento experimental}

El montaje de los moldes en cera se realiza a semejanza de los moldes de producción de "Microfusión de Aluminio, S. A." Una vez obtenidas las probetas por inyección de cera, se colocan en ramas y se montan en una estructura que posee una copa donde se vierte el metal y un filtro que evita la entrada de residuos. A continuación, se construye, por aplicación de sucesivas capas refractarias, una cáscara cerámica en torno al molde, de 5 a $8 \mathrm{~mm}$ de grosor. Tras el desencerado y la sinterización (o quemado) de la cáscara, se dispone del molde a colar.

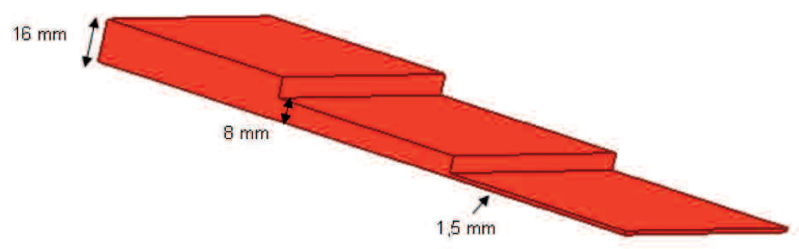

Figura 2. Dibujo de la pieza escalón.

Figure 2. Drawing of the step part. 

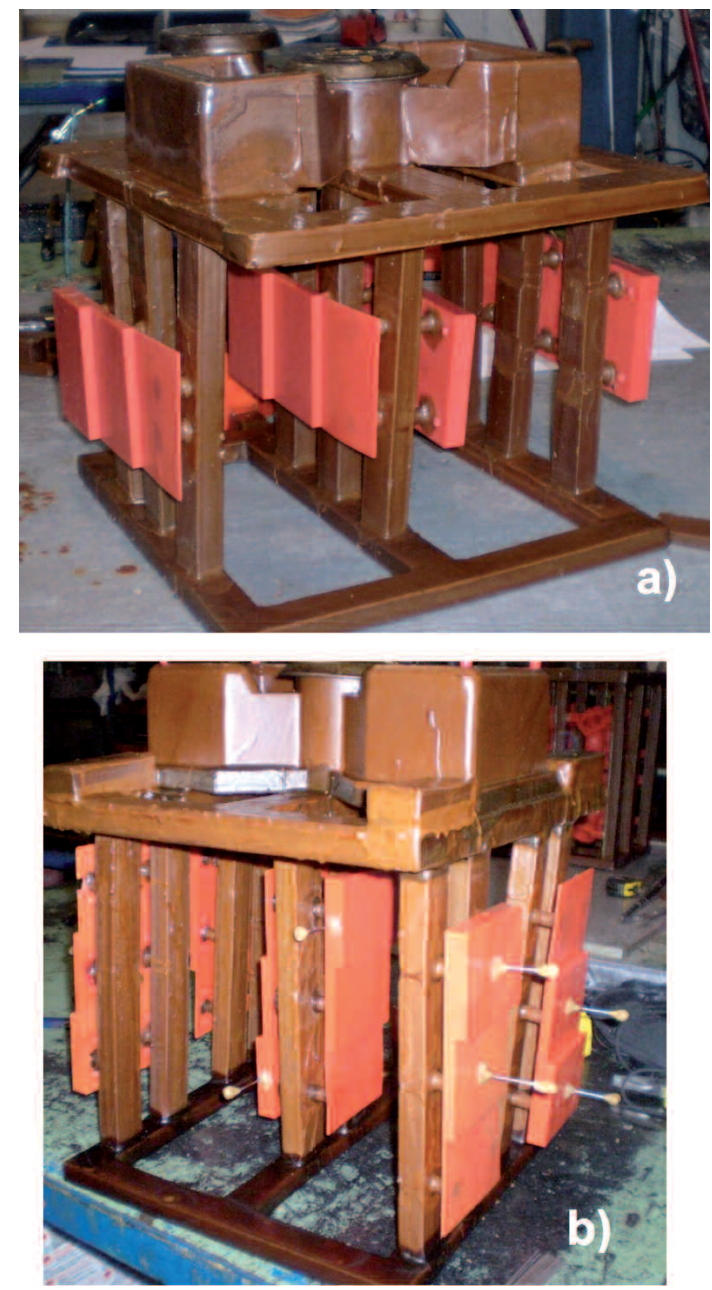

Figura 3. Moldes I (a) y II (b) en cera. En la imagen b) se muestran, también, las fundas de los termopares ya colocadas.

Figure 3. Wax moulds I (a) and II (b). On image b) location of the thermocouple covers is shown.

En ambos ensayos, se emplean termopares tipo K, de $1 \mathrm{~mm}$ de diámetro, con el fin de registrar las curvas de enfriamiento. Las fundas que protegen los termopares han sido ubicadas en el centro de la sección más grande y se han introducido hasta la mitad del grosor de cada escalón, salvo en el caso de los escalones de 1,5 mm, en los que no se han podido colocar debido a las dificultades que, una sección tan fina, supone. En los peldaños interiores, los termopares se introducen desde el canto hasta una profundidad de unos pocos milímetros.

Ambos moldes son quemados a $850^{\circ} \mathrm{C}$. En el momento de la colada, el molde se extrae del horno a una temperatura de $700{ }^{\circ} \mathrm{C}$, mientras que el caldo metálico se halla en torno a los $737^{\circ} \mathrm{C}$ (en horno). El llenado tiene lugar desde la parte inferior hacia la superior. Una vez completo, se deja enfriar al aire, al mismo tiempo que la temperatura indicada por los termopares es registrada gracias a un captador de datos, a una frecuencia de 10 puntos/segundo.

Las aleaciones estudiadas encajan en la calidad A 357 y se ha realizado con un $80 \%$ de retorno interno y $20 \%$ de lingote. El caldo es afinado con pastillas de boro, modificado con sodio y desgasificado mediante barboteo de argon durante 15-20 min. La composición química de ambos ensayos viene dada en la tabla I.

Las piezas han sido inspeccionadas por rayos $\mathrm{X}$ con el fin de valorar el grado de porosidad interna. De cada uno de los escalones se toma, además, una muestra para análisis metalográfico, proveniente de las proximidades de medida del termopar.

\section{RESULTADOS Y DISCUSIÓN}

\subsection{Análisis radiográfico}

El análisis radiográfico permite conocer la distribución de la microporosidad dentro de la pieza, es decir, su sanidad interna. En el caso de las probetas del molde I, todas se llenan de la misma manera: el flujo entra desde el canto y llena la cavidad de los tres escalones a la vez (Fig. 4 a)). Ninguno de ellos presenta rechupes internos.

En el ensayo II, por el contrario, se observan zonas de rechupes pequeños y dispersos, independientemente de la orientación de la probeta. Además, cuando el llenado se produce desde el escalón grueso, se aprecia, en alguno de los escalones intermedios, rechupe de contracción esponjoso, que indica que el escalón grueso se ve alimentado por el metal, aún líquido, del escalón intermedio. Por este motivo, se determina la necesidad de llenar desde la sección fina, tal y como se indica en la figura 4 b).

Tabla I. Composición química del aluminio, en cada una de las coladas

Table I. Chemical composition of aluminum in each of the trials

\begin{tabular}{ccccccc}
\hline & \multicolumn{5}{c}{$\begin{array}{c}\text { Composición química } \\
\text { (\% en peso) }\end{array}$} \\
I Molde & \multicolumn{6}{c}{ (\% en } \\
\cline { 2 - 7 } & $\mathbf{S i}$ & $\mathbf{M g}$ & $\mathrm{Ti}$ & $\mathbf{F e}$ & $\mathbf{C u}$ & $\mathbf{M n}$ \\
\hline I & 6,64 & 0,58 & 0,11 & 0,16 & 0,01 & $<0,01$ \\
II & 6,75 & 0,57 & 0,11 & 0,16 & 0,01 & $<0,01$ \\
\hline
\end{tabular}



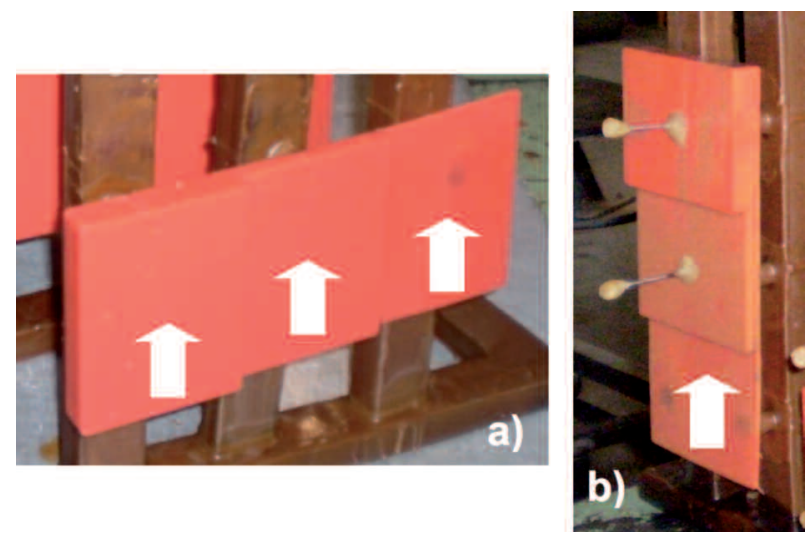

Figura 4. Orden de llenado de las piezas en cada uno de los ensayos. En (b), la posición invertida de la probeta escalón facilita la aparición de rechupes.

Figure 4. Filling of the parts in each of the trials. On (b), the inverted position of the step-part makes it prone to shrinkage porosity.

La curva de enfriamiento y la microestructura de cada escalón del ensayo II se han visto condicionadas, por tanto, por su llenado y alimentación, dificultando el análisis y comparación entre los resultados, para los diferentes grosores y las posiciones de las probetas. Teniendo en cuenta que los mejores resultados se han obtenido para el ensayo I, en el que todos los escalones han seguido el mismo orden de llenado, el análisis de estructura y de las curvas de solidificación se centrará en este ensayo.

\subsection{Curvas de enfriamiento}

En el ensayo I se registran, correctamente, las medidas de cuatro de los termopares introducidos. En la figura 5, se muestra su ubicación y en la figura 6, las curvas registradas, que se analizan a continuación.

A pesar de que el termopar ubicado en la posición TC4 $(16 \mathrm{~mm})$ no entrara hasta el fondo de la funda del termopar, al compararlo con los valores registrados por el TC1 se observan similares tiempos de inicio de solidificación del Al-Si y, también, de longitud del plateau eutéctico, por lo que se puede considerar que ambas zonas han sufrido similar velocidad de enfriamiento. La diferencia de $15^{\circ} \mathrm{C}$ de temperatura, en el plateau eutéctico del termopar TC4, se considera que ha sido causada por la falta de contacto de la punta del termopar con el fondo de la funda. Así, la mayor o menor proximidad al filtro no parece afectar a su comportamiento en el enfriamiento. Teniendo en cuenta la igualdad de tiempos, mencionada previamente, entre el TC4 y el TC1,
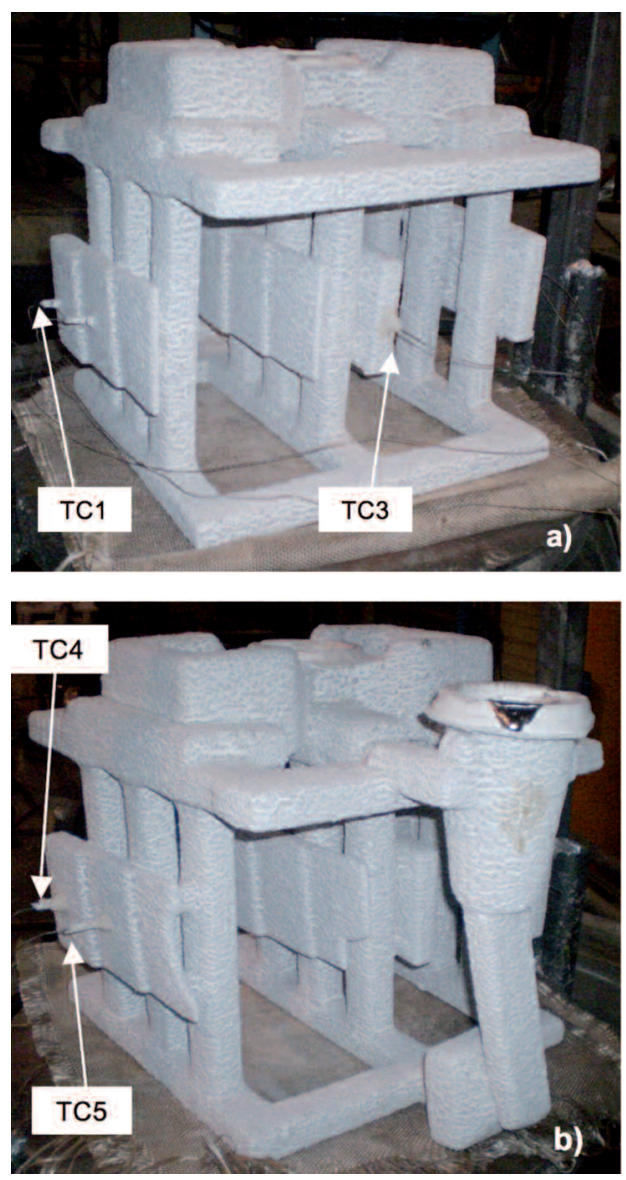

Figura 5. Molde I ya colado. TC1 y TC4 se sitúan en escalones gruesos (16 mm) y exteriores; TC3, en uno grueso interior $y$, finalmente, TC5, en un escalón exterior intermedio $(8 \mathrm{~mm})$.

Figure 5. Mould I already cast. TC1 and TC4 are located in the thick (16mm) outer steps; TC3, in a thick inner one and TC5 in an intermediate (8mm) outer step.

parece razonable comparar los resultados de TC1 $(16 \mathrm{~mm})$ y TC5 $(8 \mathrm{~mm})$ como si perteneciesen al mismo escalón. El resultado es el esperado: el escalón intermedio (TC5) se enfría antes que el grueso. Finalmente, en cuanto al TC3, introducido desde la sección lateral del escalón de $16 \mathrm{~mm}$ hasta una profundidad de unos pocos milímetros, se observa que el enfriamiento es bastante más lento. Tal efecto se atribuye al hecho de que se halle en la parte interior del molde, rodeado de elementos a alta temperatura, que lo mantienen más tiempo caliente.

Si bien en los escalones finos no se ha registrado la curva de enfriamiento, la microestructura es más fina y el tamaño de las dendritas y el espaciado interdendrítico es menor que en el resto de escalones, tal y como se puede comparar en la figura 7 a) y b). 


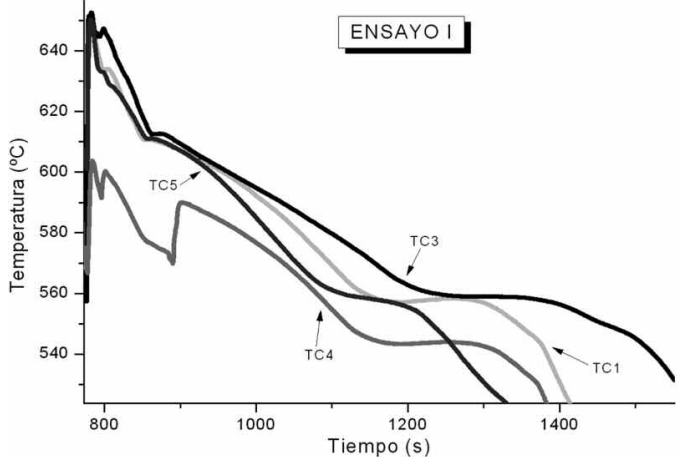

Figura 6. Curvas de enfriamiento registradas en el ensayo I. TC1: escalón de $16 \mathrm{~mm}$, exterior, cerca del filtro. TC3: escalón de $16 \mathrm{~mm}$ interior. TC4: escalón de $16 \mathrm{~mm}$, exterior, lejos del filtro. TC5: escalón de $8 \mathrm{~mm}$, exterior.

Figure 6. Cooling curves recorded on trial I. TC1: $16 \mathrm{~mm}$ outer step, close to the filter. TC3: $16 \mathrm{~mm}$ inner step. TC4: $16 \mathrm{~mm}$ outer step, far from the filter. TC5: $8 \mathrm{~mm}$ outer step.
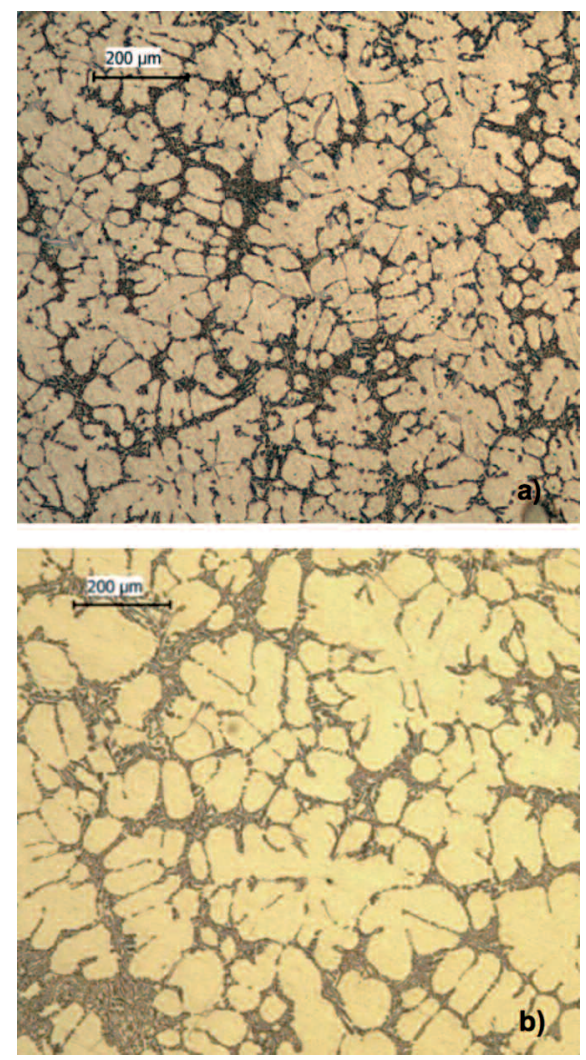

Figura 7. Microestructura del escalón de 1,5 mm (a) frente a la del escalón de $16 \mathrm{~mm}$, donde se halla TC1 (b). La diferencia en el espaciado interdendrítico es evidente.

Figure 7. Microstructure of the $1.5 \mathrm{~mm}$ step (a) compared to $16 \mathrm{~mm}$ step where TC1 is situated (b). Difference in interdentritic spacing is obvious.

\subsection{Grado de modificado}

El grado de modificado se valora de manera cuantitativa, midiendo el tamaño de las partículas de silicio mediante técnicas de análisis de imagen. Si el modificado es correcto, la estructura fibrosa del silicio se traduce en partículas pequeñas y circulares en el plano; si, por el contrario, el modificado no es bueno, se observa una geometría acicular o laminar y de mayor tamaño. En la mayoría de las muestras existe una convivencia de tipos de modificado, pero los resultados expuestos muestran la estructura representativa, en cada caso. Ejemplos de las estructuras obtenidas se muestran en la figura 8. Para cada escalón, se toman entre 3 y 5 imágenes.

Seguidamente, se muestra el porcentaje acumulado de número de partículas, en función del área de las mismas, en dos casos: escalones de 8 y de $16 \mathrm{~mm}$, de la Prueba 1.
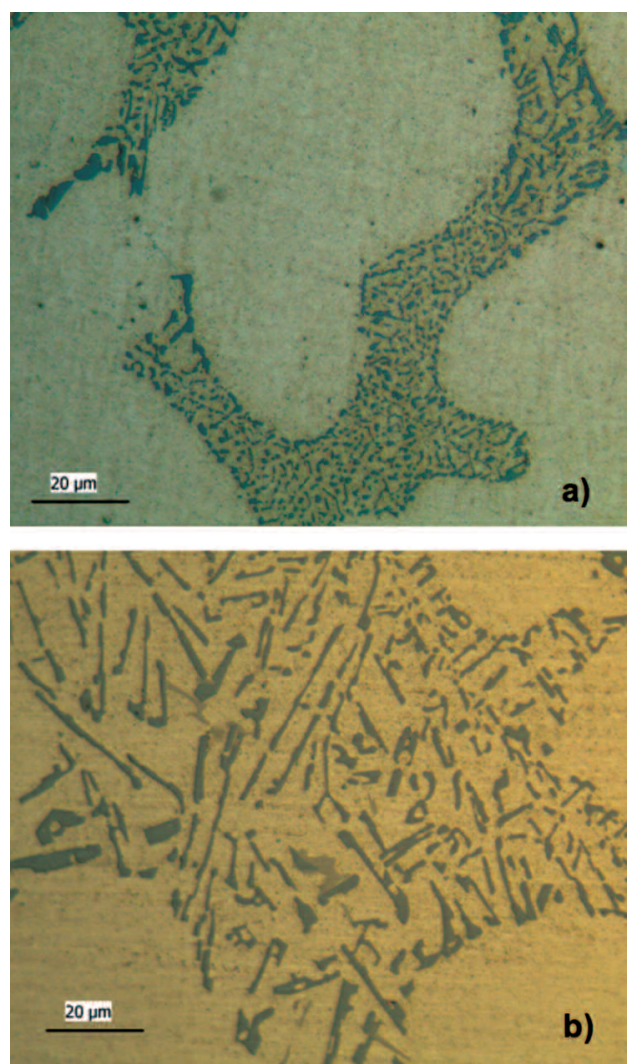

Figura 8. Estructura de silicio eutéctico correctamente modificado, observada en uno de los escalones finos (a) frente a la estructura laminar (submodificada) obtenida en uno de los escalones gruesos (b).

Figure 8. Structure of well modified eutectic Si found in one of the thin steps (a) compared to the lamellar (submodified) structure obtained in one of the thick steps (b). 
En la figura 9 se muestra el caso de los escalones de $16 \mathrm{~mm}$. Como se puede observar, el hecho de que la probeta se halle en la parte exterior o interior del molde es determinante: a pesar de hallarse lejos del punto de llenado, la muestra de la probeta en el interior (TC3), presenta un tamaño de partículas de silicio similar a la que se halla cerca del filtro y es exterior (TC1). Ambos grados de modificado son inferiores al del escalón exterior, lejos del filtro TC4. Tal hecho se observa en sus microestructuras (Fig. 10 a) y b)).

En la figura 11, donde se comparan los resultados para los escalones de $8 \mathrm{~mm}$, todos ellos, con la misma cercanía al filtro, se confirma que la disposición externa o interna de la probeta en el molde resulta clave. Así, las gráficas de los escalones exteriores son, prácticamente, equivalentes y el tamaño de sus partículas, inferior al del silicio del escalón interior, puesto que al no estar rodeadas de más zonas calientes enfrían antes.

\section{CONCLUSIONES}

Con el objetivo de caracterizar la solidificación de las piezas de aluminio moldeadas a la cera perdida y establecer una equivalencia con los resultados aportados por los métodos de análisis térmico, se requiere un molde que dé cobertura a la variedad de espesores y disposición de piezas en la industria de microfusión. Para ello, se han realizado varias pruebas cuyos resultados permiten determinar el diseño del molde más adecuado. En resumen, se ha observado que:

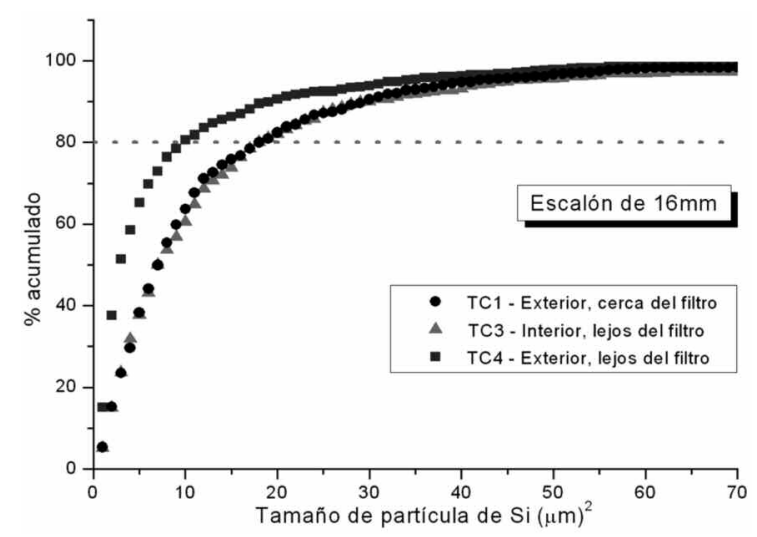

Figura 9. Porcentaje acumulado de número de partículas en función de su área, para los escalones de $16 \mathrm{~mm}$. La línea del $80 \%$ se ha tomado como referencia.

Figure 9. Cumulative percentage of the number of particles versus their area for $16 \mathrm{~mm}$ steps. The line at $80 \%$ has been taken as reference.
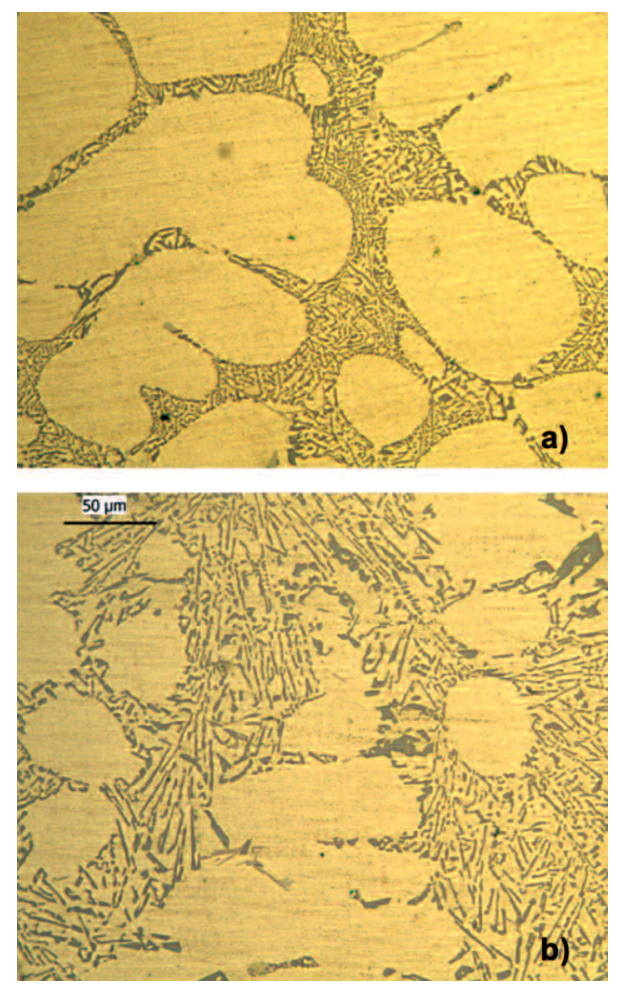

Figura 10. Microestructura en torno al termopar TC4, con un eutéctico más fibroso (a) frente a la observada en TC3 (b), donde el silicio se presenta más laminar.

Figure 10. Microstructure in the surroundings of thermocouple TC4 with a fibrous eutectic (a) compared to the microstructure obtained around TC3 (b), where silicon shows up in lamellar form.

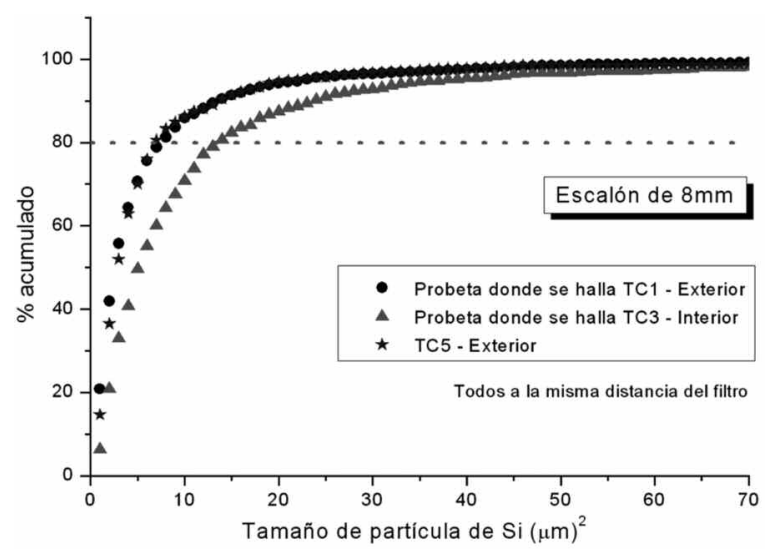

Figura 11. Porcentaje acumulado del número de partículas en función del área de las mismas, para los escalones de $8 \mathrm{~mm}$.

Figure 11. Cumulative percentage of the number of particles versus their area for $8 \mathrm{~mm}$ steps. 
- La probeta escalonada ha de ir en posición horizontal para evitar la aparición de rechupes.

- La velocidad de enfriamiento es inversamente proporcional al grosor de la pieza.

- La cercanía del escalón grueso al filtro afecta al grado de modificado.

- Los mejores resultados de grado de modificado y velocidad de enfriamiento se obtienen en las probetas exteriores, pues enfrían antes.

Así, se establece la necesidad de crear un nuevo utillaje para la probeta escalonada, añadiendo un espesor intermedio más, para ampliar la variedad y aumentando el grosor del escalón más fino, pues 1,5 $\mathrm{mm}$ resulta delicado en la manipulación. Resultado: probeta de cuatro escalones, de 2, 4, 8 y $16 \mathrm{~mm}$.

Se concluye, también, que una única probeta escalonada por molde es suficiente y se intentan conseguir condiciones extremas de solidificación: el escalón más fino se sitúa lejos del filtro, mientras que el más grueso se coloca cerca, consiguiendo las mayores y menores velocidades de enfriamiento, respectivamente. Además, dado que la presencia de espesores tan grandes como $16 \mathrm{~mm}$ no es habitual, se da preferencia a la obtención de mejores grados de modificado en espesores finos.

Asimismo, se opta por incluir en el molde probetas separadas de tracción (6 mm de diámetro) y probetas integradas cilíndricas ( $10 \mathrm{~mm}$ de diámetro, para ensayar tras su mecanizado), cuyo empleo es habitual como medio de control en las coladas. Con ello, se busca una relación directa entre los parámetros de análisis térmico que definen y controlan el estado del caldo y las propiedades mecánicas finales. yb).

El molde resultante se muestra en la figura 12 a)

\section{REFERENCIAS}

[1] A. Loizaga, A. Niklas, A.I. Fernández-Calvo y J. Lacaze, Int. J. Cast Metals Res., 22 (2009) 345-352.

[2] G. K. Sigworth y T. A. Kuhn, AFS Trans. 07067 (2007) 31-40.
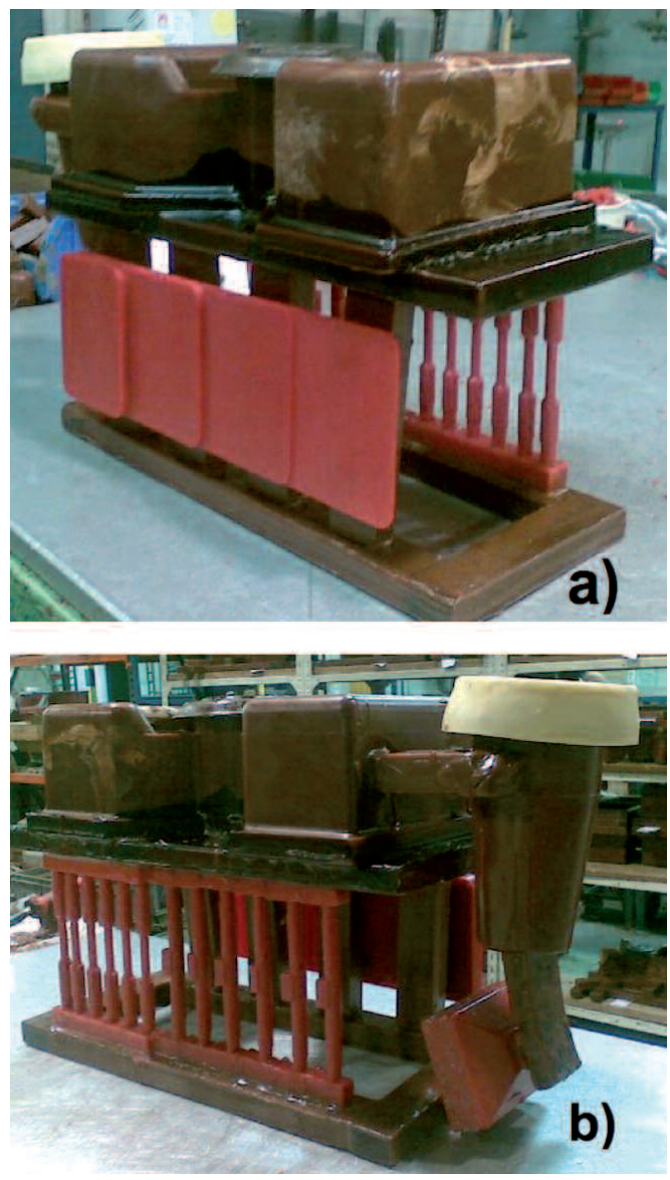

Figura 12. Molde creado como resultado de las pruebas y análisis expuestos en el presente trabajo.

Figure 12. Mould designed as a result of the trials and analysis exposed on the present work.

[3] S. Seifeddine, I. L. Svensson, 67th World Foundry Congress, Paper 200, Harrogate, Inglaterra, 2006.

[4] F. Chiesa, K. Durand y G. Morin, 11th World Conf. Investment Casting, Paper WC 11.13, Edinburgh, Inglaterra, 2004.

[5] J. Bäckman y I. L. Svensson, Research report, School of Engineering, Jönköping University, Sweden, 1999. 